\section{Edentulism trends among middle-aged and older adults in the United States: comparison of five racial/ethnic groups}

Wu B, Liang J, Plassman BL, Remle C, Luo X. Edentulism trends among middle-aged and older adults in the United States: comparison of five racial/ethnic groups. Community Dent Oral Epidemiol 2012; 40: 145-153. (C) 2011 John Wiley \& Sons A/S

Abstract - Objectives: This study examined edentulism trends among adults aged 50 and above in five ethnic groups in the United States: Asians, African Americans, Hispanics, Native Americans, and non-Hispanic Caucasians. Methods: Data came from the National Health Interview Surveys between 1999 and 2008. Respondents included 616 Native Americans, 2,666 Asians, 15,295 African Americans, 13,068 Hispanics, and 86,755 Caucasians. Results: In 2008, Native Americans had the highest predicated rate of edentulism (23.98\%), followed by African Americans (19.39\%), Caucasians (16.90\%), Asians (14.22\%), and Hispanics (14.18\%). Overall, there was a significant downward trend in edentulism rates between 1999 and 2008 (OR $=0.97,95 \%$ CI: 0.96, 0.98). However, compared with Caucasians, Native Americans showed a significantly less decline of edentulism during this period ( $\mathrm{OR}=1.10,95 \% \mathrm{CI}: 1.02,1.19$ ). Conclusions: While there was a downward trend in edentulism between 1999 and 2008, significant variations existed across racial/ethnic groups. Innovative public health programs and services are essential to prevent oral health diseases and conditions for minority populations who lack access to adequate dental care. Additionally, given the increasing numbers of adults retaining their natural teeth, interventions designed to assist individuals in maintaining healthy teeth becomes more critical.
Bei $\mathrm{Wu}^{1}$, Jersey Liang ${ }^{2}$,

Brenda L. Plassman ${ }^{3}$, Corey Remle ${ }^{4}$ and Xiao Luo $^{5}$

${ }^{1}$ School of Nursing, Duke University, Durham, NC, USA, ${ }^{2}$ School of Public Health, University of Michigan, Ann Arbor, MI, USA, ${ }^{3}$ Psychiatry and Behavioral Sciences, Duke University, Durham, NC, USA ${ }^{4}$ Sociology, Wake Forest University, Winston Salem, NC, USA, ${ }^{5}$ Gerontology, University of North Carolina, Greensboro, NC, USA

Key words: disparities; edentulism; older adults; trend analysis

Bei Wu, School of Nursing, Duke University, 307 Trent Drive, DUMC 3322, Durham, NC 277170, USA

Tel.: +919-684-7534

Fax: +919-681-8899

e-mail: bei.wu@duke.edu

Submitted 17 August 2010; accepted 8 August 2011
Edentulism, or complete tooth loss, is one of the most important indicators of oral health. Edentulism reflects both the accumulated burden of oral diseases and conditions and the results of dental extraction treatment (1). Studies suggest that edentulism significantly affects quality of life, selfesteem, and nutritional status (2-4). In economically developed countries, the trend of edentulism has declined consistently. For example, in England and Wales, the prevalence of edentulism for the adult population declined from $37 \%$ in 1968 to $12 \%$ in 1998 (5). In Australia, the prevalence of edentulism for the adult population declined from $20.5 \%$ in
1979 to $8.0 \%$ in 2002. Among older adults aged 65 and above, the reduction for males was from 59.7\% to $26.5 \%$ and for females was from $71.5 \%$ to $40.3 \%$ (1). Similarly, in the United States, the few studies available on middle-aged and older adults have shown that edentulism in these age groups has been dropping for the past several decades. One study revealed that within the period of 1971 and 2001, for those in a low socioeconomic position (SEP), the prevalence of edentulism declined from $50 \%$ to $32 \%$ in adults age 55-64 and 58-43\% in age 65-74; the comparable declines for these age groups for individuals in a high SEP were $22-6 \%$ and $30-9 \%$, 
respectively (6). A report conducted by the National Centers for Health Statistics using the National Health and Nutrition Surveys of 1988-1994 (NHANES III) and NHANES 1999-2004 found that the prevalence of edentulism declined in the United States over these two-time periods from $34 \%$ to $27 \%$ among adults aged 65 and older over (7).

Minority elders have been identified as a key demographic group at greatest risk for edentulism (8). Black elders, in particular, have higher rates of edentulism than non-Hispanic Caucasians and Mexican Americans $(7,9,10)$. But one study found that the rates of edentulism were declining even though they were still higher than other ethnic groups (7). This study reported that the rates of edentulism for Black elders declined from $38 \%$ in 1988-1994 to 33\% in 1999-2004 (7). For Caucasians, the percentages were much lower: $34 \%$ in 1988 1994 and 26\% in 1999-2004. By comparison, Mexican American adults had even lower edentulism rates $(27 \%$ and $24 \%)$.

Information regarding edentulism for Asian Americans and Native Americans is very limited. A recent report determined that $21 \%$ of Asian Americans aged 65 and over had lost all teeth compared to $25 \%$ of Caucasians. Asians also had the lowest percentage of edentulism compared with other minority groups (9). There are no oral health trend studies that compare Native Americans to other ethnic groups. However, the third Oral Health Survey conducted by the Indian Health Service in 1999 found that 21\% of Native American adults aged 55 and older were edentulous, representing a decrease of $5 \%$ over 15 years (11).

Although the few studies cited earlier provide useful information on the prevalence of oral health for major demographic subgroups such as Caucasians, African Americans, and Hispanics, the analyses of trends in oral health disparities have been cursory only providing crude prevalence of edentulism, and have rarely included formal statistical tests adjusting for population heterogeneity that could potentially contribute to the impact of race/ethnicity on oral health outcomes. The limited evidence suggesting improvements in oral health among the adult population in the United States has left unanswered the question of whether these recent improvements have been experienced broadly across all racial and ethnic groups. More studies are required to identify factors that contribute to oral health disparities and to provide a broader and more accurate picture of edentulism trends among older adults.
The present study examined the trend of edentulism among adults aged 50 and above in five ethnic groups: Asians, African Americans, Hispanics, Native Americans, and non-Hispanic Caucasians. The uniqueness of the study was built on the following factors: (i) to provide national estimates on the rate of edentulism for five major racial/ethnic groups in the United States, including Asians and Native Americans for whom very limited information was previously available; (ii) to compare edentulism trends across the racial and ethnic groups; and (iii) to investigate a comprehensive array of factors that could contribute to improvements in edentulism across populations over time.

\section{Methods}

\section{Data sources and sample}

This study used the National Health Interview Survey (NHIS) which is a cross-sectional household interview survey conducted annually. The main objective of the NHIS is to monitor the health of the United States population through the collection and analysis of data on a broad range of health topics. The multistage area probability design provides a representative sample of the civilian noninstitutionalized population residing in the United States at the time of the interviews. We aggregated ten waves of NHIS data collected from 1999 to 2008. Eligible respondents were those aged 50 and above who completed the question on tooth loss. The sample included 616 Native Americans, 2,666 Asians, 15,295 African Americans, 13,068 Hispanics, and 86,755 non-Hispanic Caucasians.

\section{Measures}

Dependent variable. Self-reported responses to a question about whether the individual had lost all upper and lower natural teeth were used to determine edentulism. Edentulism was coded as 1 and otherwise as 0 .

Independent variables. Ethnicity was measured using dichotomous variables for Native Americans, Asian Americans, non-Hispanic African Americans, Hispanics, and non-Hispanic Caucasians. Time ranged from 1 to 10 as it was measured by the number of years of the NIHS data included in the study beginning in 1999 .

Covariates. The sociodemographic variables included age measured in years (50-85, age was top coded as 85$)$, gender ( $1=$ female, $0=$ male), and 
marital status $(1=$ married/living with partner, $0=$ otherwise). Socioeconomic status was measured by educational level $(1=1$ st -11 th grade, $2=$ high school graduate, $3=$ some college, $4=$ bachelor's degree, $5=$ graduate or professional).

Health status included self-reported medical conditions, functional status, and memory problems. The medical conditions were measured by asking the respondent whether he/she had been told by a doctor that he/she had a heart attack, coronary heart disease, diabetes, hypertension, emphysema, asthma, and/or chronic bronchitis. For the analyses, the variable 'lung disease' was created by collapsing emphysema, asthma, and chronic bronchitis into one medical condition variable. Each medical condition was treated as a dichotomous variable. Functional status was measured by reported limitations in activities of daily living (ADLs) and limitations in their ability to complete routine activities (IADLs). The Activities of Daily Living scale (ADLs) was a sum measure of six self-reported needed helps in personal care including eating, dressing, bathing, using toilet, getting around in the home, and getting in and out of bed, with a range of 0-6. An Instrumental Activities of Daily Living (IADL) was defined as a dichotomous measure whether respondents needed help with routine needs $(1=$ needed help with routine needs, $0=$ otherwise). No detailed information of IADL was included in the NHIS survey. Memory problems were measured by asking whether the respondent was limited by difficulty remembering ( 1 = difficulty remembering; 0 = otherwise). Health behavior was measured by smoking status ( $1=$ current smoker, $0=$ otherwise).

\section{Data analysis}

We used SAS 9.1 (SAS Institute Inc., Cary, NC, USA) for all analyses. The General Linear Model (GLM) procedure was used to test the differences in sample characteristics across the five ethnic groups and between groups. As the edentulism variable is dichotomous, the predicted probability of the edentulism rate was calculated using logistic regression adjusting for time, race/ethnicity, sociodemographic characteristics, and level of education. To estimate the rate of edentulism, we summed the value of the probability of edentulism for all respondents within each racial/ethnic group per year and divided the sum total by the number of participants in the corresponding group.
Weighted logistic regression was performed with edentulism as the outcome variable. For the analyses, time was recoded to be centered around the mean to reduce multicollinearity when testing interactions between time and race/ethnicity. A hierarchical block design was used in multivariate analyses to determine the separate contributions of all variables included in the study. The first step included time and race/ethnicity. In the second step, the sociodemographic variables and education were added. The third step added smoking and health status, which was measured by functional status, medical conditions, and memory problems. To test the change in edentulism by race/ethnicity over time, the full model also included interaction terms for time by race/ethnicity.

\section{Results}

\section{Sample characteristics}

Sample characteristics were significantly different across racial/ethnic groups (Table 1). Overall, compared with Caucasian respondents, African Americans, Hispanics, and Native Americans had lower levels of education. These individuals also reported higher rates of smoking and poorer health status based on their functional status and rate of memory problems and diabetes.

\section{Edentulism across racial/ethnic groups}

For the past 10 years, there was an overall declining trend of edentulism for all racial and ethnic groups, except for Native Americans (Table 2). Table 2 presents the predicted rate of edentuliusm adjusting for time, sociodemographic characteristics, and level of education. In 2008, Native Americans had the highest rate of edentulism (23.98\%), followed by African Americans (19.39\%), Caucasians (16.90\%), Asians (14.22\%), and Hispanics $(14.18 \%)$. Figure 1 presents the trend of predicted rate of edentulism adjusting for time, sociodemographic characteristics, and level of education.

Since 2004, the rate of edentulism has been fairly stable for all groups, except for Native Americans. Native Americans showed a higher rate of edentulism overall for this 10-year period. The rate of edentulism for Native Americans was also the most variable, possibly due to the relatively small sample size compared with the other racial and ethnic groups. 
Wu et al.

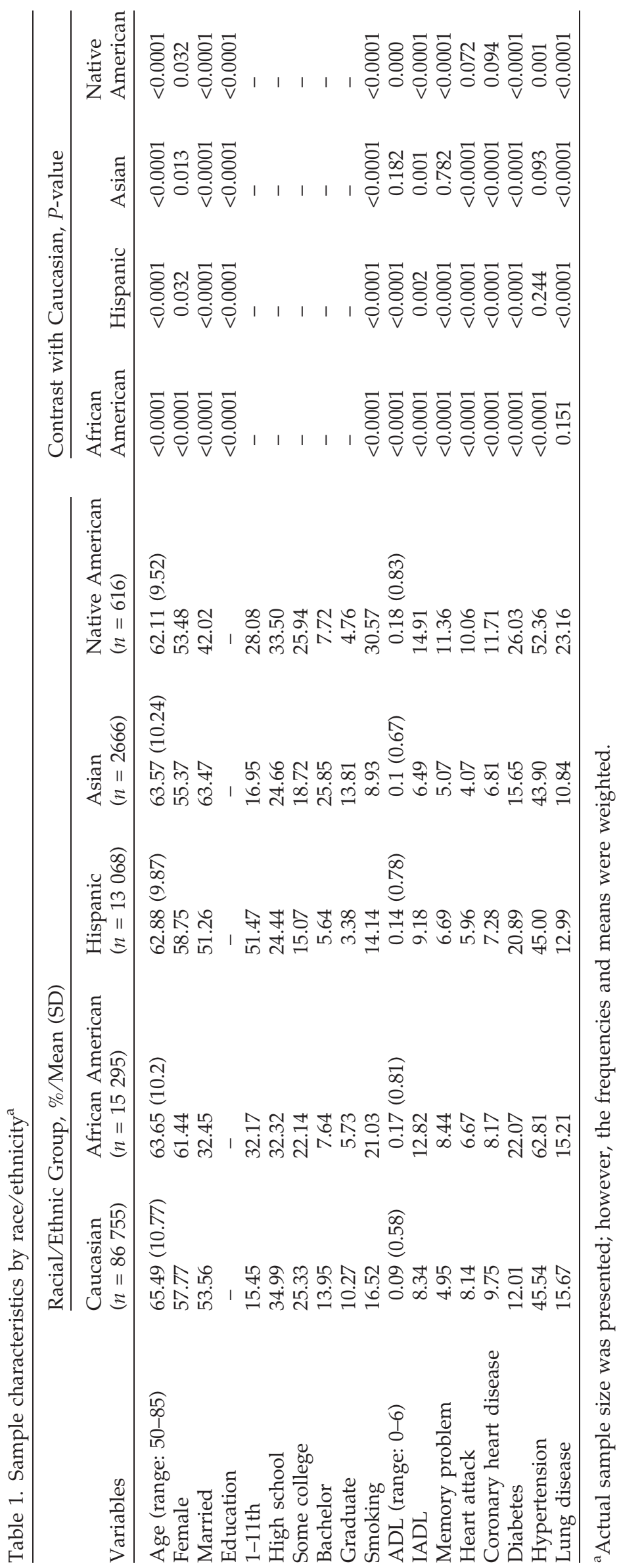


Table 2. Trend of edentulism by racial/ethnic groups (1999-2008) (\%; Weighted $)^{\mathrm{a}}$

\begin{tabular}{llllll}
\hline Time & Caucasian & African American & Hispanic & Asian American & Native American \\
\hline 1999 & 21.49 & 24.62 & 17.78 & 17.04 & 33.20 \\
2000 & 21.18 & 23.74 & 17.60 & 13.54 & 34.02 \\
2001 & 20.20 & 23.02 & 17.71 & 11.88 & 31.78 \\
2002 & 19.77 & 22.42 & 16.68 & 13.55 & 29.72 \\
2003 & 18.90 & 21.78 & 16.21 & 15.88 & 29.67 \\
2004 & 18.80 & 20.60 & 15.44 & 14.09 & 28.12 \\
2005 & 17.98 & 20.65 & 15.13 & 13.57 & 24.72 \\
2006 & 17.58 & 20.62 & 15.20 & 15.26 & 30.18 \\
2007 & 17.05 & 19.58 & 14.74 & 14.08 & 27.07 \\
2008 & 16.90 & 19.39 & 14.18 & 14.22 & 23.98 \\
\hline
\end{tabular}

${ }^{a}$ The predicted rates of edentulism were calculated by weighted analysis adjusting for time, race/ethnicity, sociodemographic characteristics, and level of education.

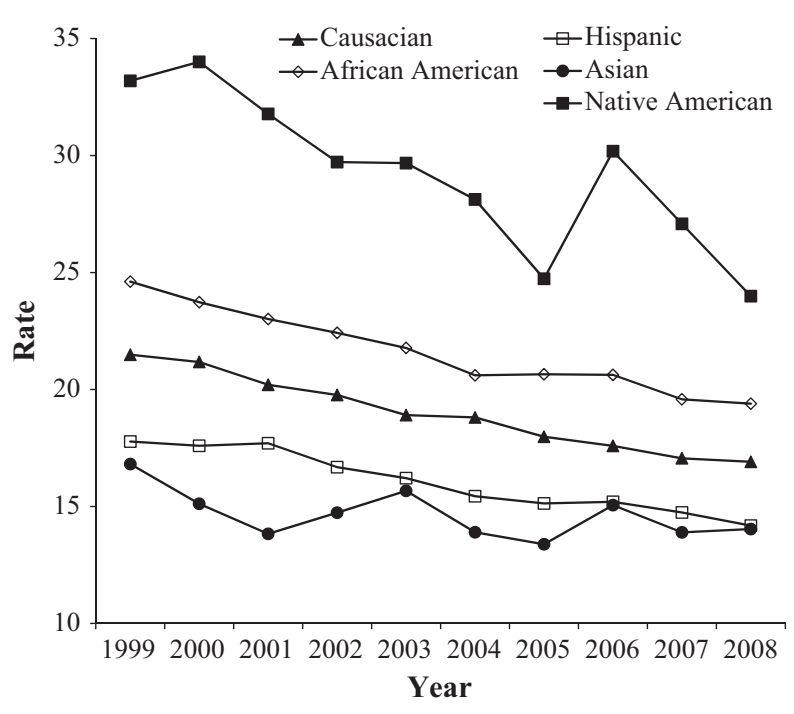

Fig. 1. Predicted rate of edentulism.

Logistic regression analysis results showed that overall, there was a significant downward trend of edentulism between 1999 and $2008(\mathrm{OR}=0.97,95 \%$ CI: 0.96, 0.98) (Table 3). Over the 10-year period (1999-2008), there was about 3\% decrease in odds of being edentulous each year from the previous year. The interaction term of time and race/ethnicity showed that compared with Caucasians, edentulism among Native Americans was less likely to decline during this period (OR $=1.10,95 \%$ CI: 1.02 , 1.19). In contrast, the declining trend of edentulism did not differ among Asian Americans, African Americans, or Hispanics compared to the rate of decline among Caucasians.

In models without covariates, African Americans and Native Americans had a higher likelihood of being edentulous than Caucasians, while the likelihood was lower for Hispanics and Asians (Table 3, Model 1). However, after controlling for demographic characteristics, socioeconomic status, medical conditions, functional status, and health behavior, compared with Caucasian respondents, Hispanics (OR $=0.61,95 \%$ CI: 0.56, 0.65) and African Americans (OR $=0.91,95 \%$ CI: 0.85, 0.97) were less likely to have lost all teeth (Table 3, Model 4). Asians showed a tendency toward a lower risk of edentulism than Caucasians, but the association was not significant $(\mathrm{OR}=1.02,95 \% \mathrm{CI}$ : $0.86,1.23)$. In contrast, Native Americans were more likely to be edentulous compared with Caucasians (OR $=1.36,95 \%$ CI: $1.09,1.69)$. Years of education, age, and current smoking were the covariates with the greatest impact on edentulism.

\section{Discussion}

This is the first study to provide national estimates for the rate of edentulism and associated trends over time for five major ethnic groups in the United States simultaneously: Native Americans, Asian Americans, African Americans, Hispanics, and non-Hispanic Caucasians. Significant disparities in edentulism exist across these ethnic groups. Relative to Caucasians, African Americans, and Native Americans had a higher rate of edentulism, whereas the rate of edentulism was lower among Hispanics and Asians. After controlling for covariates, African Americans and Hispanics were less likely to be edentulous than Caucasian respondents, while Native Americans were still more likely to be edentulous. In contrast, when covariates were included in the models, no significant differences were found between Asian Americans and Caucasians in edentulous rates. Overall, there was a significant downward trend in edentulism rates between 1999 and 2008; however, oral health disparities, as measured by rates of edentulism, 
Wu et al.

Table 3. Logistic regression on likelihood of being edentulism

\begin{tabular}{lllll}
\hline Variables & Model 1 & Model 2 & Model 3 & Model 4 \\
\hline Time & $0.97(0.96,0.97)^{* * *}$ & $0.98(0.97,0.99)^{* * *}$ & $0.97(0.97,0.98)^{* * *}$ & $0.97(0.96,0.98)^{* * * *}$ \\
African Americans & $1.18(1.11,1.25)^{* * *}$ & $0.95(0.89,1.01)$ & $0.91(0.86,0.97)^{* *}$ & $0.91(0.85,0.97)^{* * *}$ \\
Hispanics & $0.82(0.77,0.88)^{* * *}$ & $0.57(0.52,0.61)^{* * *}$ & $0.61(0.56,0.65)^{* * *}$ & $0.61(0.56,0.65)^{* * *}$ \\
Asians & $0.72(0.63,0.83)^{* * *}$ & $0.87(0.74,1.03)$ & $0.98(0.82,1.17)$ & $0.98(0.83,1.17)$ \\
Native Americans & $1.85(1.51,2.25)^{* * *}$ & $1.73(1.40,2.14)^{* * *}$ & $1.46(1.17,1.81)^{* *}$ & $1.36(1.09,1.69)^{* * *}$ \\
Age & & $1.05(1.05,1.05)^{* * *}$ & $1.05(1.05,1.06)^{* * *}$ & $1.05(1.05,1.06)^{* * * *}$ \\
Female & & $0.88(0.85,0.92)^{* * *}$ & $0.95(0.91,0.99)^{* *}$ & $0.95(0.91,0.99)^{* *}$ \\
Married & & $0.77(0.73,0.80)^{* * *}$ & $0.84(0.81,0.88)^{* * *}$ & $0.84(0.81,0.88)^{* * *}$ \\
Education & & $0.53(0.52,0.54)^{* * *}$ & $0.56(0.55,0.58)^{* * *}$ & $0.56(0.55,0.58)^{* * * *}$ \\
Smoking & & $2.32(2.20,2.45)^{* * *}$ & $2.32(2.20,2.45)^{* * * *}$ \\
ADL & & & $1.01(0.98,1.04)^{* *}$ & $1.01(0.98,1.04)$ \\
IADL & & & $1.31(1.21,1.41)^{* * *}$ & $1.31(1.21,1.41)^{* * * *}$ \\
Memory problem & & & $1.14(1.05,1.25)^{* *}$ & $1.14(1.05,1.25)^{* * *}$ \\
Heart attack & & $1.31(1.21,1.42)^{* * *}$ & $1.31(1.21,1.42)^{* * *}$ \\
Coronary heart disease & & & $1.09(1.01,1.17)^{* *}$ & $1.09(1.01,1.17)^{*}$ \\
Diabetes & & $1.45(1.38,1.52)^{* * *}$ & $1.45(1.38,1.52)^{* * *}$ \\
Hypertension & & $1.12(1.07,1.16)^{* * *}$ & $1.12(1.07,1.16)^{* * * *}$ \\
Lung disease & & & $1.42(1.35,1.49)^{* * *}$ & $1.42(1.35,1.49)^{* * * *}$ \\
Time $\times$ African Americans & & & & $1.02(1.00,1.04)$ \\
Time $\times$ Hispanics & & & $1.00(0.98,1.02)$ \\
Time $\times$ Asians & & & $0.99(0.93,1.06)$ \\
Time $\times$ Native Americans & & & $1.10(1.02,1.19)^{* *}$ \\
\hline
\end{tabular}

*Significant at 0.05 level, **significant at 0.01 level, and ***significant at 0.001 level.

Model 1: Time and race/ethnicity only; Model 2: adding sociodemographic characteristics and level of education; Model 3: adding variables on smoking and health conditions; and Model 4: adding interactions between time and race/ethnicity.

increased among Native Americans over time compared with Caucasians.

We also expanded upon previous work by adjusting many potential confounding factors and found that racial/ethnic differences remained net these other factors. The improvement in tooth retention was not equally distributed across the five racial and ethnic groups examined in this study. Native Americans, in particular, were at a significant disadvantage. Compared with Caucasians, Native Americans were more likely to lose natural teeth over time, but the risk became smaller after controlling for individuals' SES, health behaviors, and medical conditions.

Our study found that edentulism has continued to decline across the United States during the past decade. This comprehensive study supports previous reports about edentulism among adult populations collected in earlier time periods and across selected racial/ethnic groups $(7,9,11)$. We found that current smoking and fewer years of education were two of the covariates most strongly associated with being edentulous. Others have attributed the declining edentulous rate to the decrease in smoking and the increase in years of education among more recent cohorts (6). We also found that selected medical conditions were associated with edentulism; these were generally consistent with previous research (12). Self-reported memory problems and needing assistance with routine activities were also associated with increased risk of edentulism. We do note that because the information on covariates was not collected prospectively, we cannot determine whether the factor preceded the edentulism. Many other factors for which data were not available in this study could also contribute to the decrease in the edentulous rate, such as the introduction of fluoridation through community water treatment (13) and fluoridated toothpaste and mouth rinse $(14,15)$. Health practices such as dietary supplements, and professionally applied or prescribed gel, foam, and varnish may also contribute to improved tooth retention $(13,15$, 16). Others point to advancements in dental technologies and treatment modalities, changes in patient and provider attitudes and treatment preferences (2), improved oral hygiene, and regular use of dental services $(2,17,18)$.

The edentulism rate for Native Americans from our study was much higher than the one from the 1999 Oral Health Survey of American Indian and Alaska Native Dental Patients conducted by the Indian Health Services (IHS) (11). As the IHS survey used a convenient sample of individuals 
who were patients at IHS, it may have underestimated the actual prevalence of edentulism for Native American adults $(19,20)$. Other research has shown that the significant reduction in dental caries observed in the general US population has been seen to a lesser extent in the Native American population. Further, this reduction in the number of caries among Native Americans primarily occurred in the 1980s, and did not continue into the late 1990s, resulting in a greater disparity in oral health in recent times between the general population and Native Americans (20). Our findings further demonstrate that there have been greater oral health disparities between the general population and Native Americans during the past decade.

Access to dental care is a particular challenge for Native Americans. IHS is the main federal agency to provide health care for American Indians and Alaska Natives in the United States. Recruitment and retention of dental health care professionals are a continuing and worsening problem for IHS because of low salaries, the length of commitment required, more attractive employment opportunities elsewhere, and other factors. Currently, 32\% of the full-time dental positions in IHS are vacant, an all-time high (21). IHS records also show that, although elders comprise $6 \%$ of the Native American population, they represent $<3 \%$ of the Native American dental patients seen annually across all IHS programs. Furthermore, as of 10 years ago, most older Native Americans received dental care only for the relief of symptoms, and less than onethird who sought care had a routine oral examination (19); it is unclear whether this practice has changed over the past decade. While it may be physically and financially challenging to provide adequate dental care to this population, implementation of innovative programs and services will be critically important to preventing oral health diseases and conditions. Recently, efforts have been made at the federal level to induce health care profession schools to introduce students to career opportunities offered by IHS. From a research perspective, to date, many fundamental aspects of American Indian and Native Alaskan health, including oral health, have not been systematically addressed. In late 2008, the National Institute of Dental and Craniofacial Research (NIDCR) began supporting a new oral health disparities center in Denver as Step 1 in the research process. The main thrust of this center's research will be the prevention of childhood caries or tooth decay. No specific programs have been initiated to address oral health issues for older adults in this population.

Our study found that African Americans and Hispanics were less likely to be edentulous than Caucasians after controlling for SES, health behavior, dental care utilization, and other covariates. This finding is consistent with our recent study using data from the clinical examinations from NHANES (1999-2004) (10). Some anecdotal evidence has suggested that minorities are particularly concerned about saving their teeth for esthetic reasons (22). We surmise that the level of concern may be greater for those with relatively higher SES. However, the value of saving natural teeth could also be compromised by the general lack of dental care among minority elders. Our recent study found that while African Americans and Mexican Americans were less likely to be edentulous than Caucasians, they were more likely to have a higher number of decayed teeth and fewer filled teeth. We presume this means that although African Americans and Hispanics have poor oral health, they have not had decayed teeth extracted. This finding may be due to both attitudinal and access to care issues. Further research is warranted on this issue. Given the increasing numbers of minority older adults retaining their natural teeth, the issue of maintaining healthy teeth in later life becomes more critical.

There was no sign of a widening gap in oral health disparity (i.e., edentulism) between African Americans and Caucasians in the past decade. This may reflect a beneficial effect of some recently developed programs and services in black communities in addressing oral health disparities (8). Further studies are warranted regarding other oral health indicators such as dental caries and periodontal diseases across racial/ethnic groups in the United States.

Asian Americans had a lower rate of edentulism compared with most other racial/ethnic groups in the United States. However, it may not necessarily mean that Asian Americans have better oral health compared with other racial/ethnic groups. Some anecdotal evidence from one US community study indicated that low-income Asian American elders had a higher number of teeth remaining compared with their Black, Hispanic, and Caucasian counterparts; nonetheless, they had the worst periodontal disease indicators measured by calculus and attachment loss (23). In addition, Asian American elders were least knowledgeable about oral health. Another issue worth pointing out is that the NHIS 
excluded non-English speaking Asian Americans in the survey. The majority of the non-English speaking Asian Americans have lower levels of education and income, lack access to dental care, and consequently have poorer oral health status. Therefore, the results from our study may provide an incomplete picture of the edentulism rate for Asian Americans even though they are based on a nationally representative sample.

\section{Study limitations}

There are some limitations inherent in the NHIS survey data. The sample was drawn from the community-dwelling population which excluded individuals residing in institutions (e.g. nursing homes or other long-term care facilities). Additionally, the edentulism rate was based on self-reports; however, strong agreement between self-reported and clinically assessed tooth counts has been documented in past studies $(24,25)$. The estimates of edentulism for Native Americans fluctuate across timepoints. This is most likely due to the small sample size and raises some questions about the robustness of the estimates. The small sample size also prohibits us from conducting more detailed subgroup analysis based on age, gender, and other demographic groups. The survey data lacked information on personal oral hygiene behaviors; such behaviors likely contribute to the risk of edentulism and may affect other clinical measures of oral health. Future research needs to examine subgroups within each ethnic group given minority populations are heterogeneous. It is likely other unmeasured factors such as quality of dental care and oral health knowledge play a role in edentulism $(18,26)$.

\section{Conclusions}

While there was a downward trend in edentulism rates between 1999 and 2008, significant variations existed across five major racial/ethnic groups. Innovative public health programs and services are essential to prevent oral health diseases and conditions for minority populations who lack access to adequate dental care. Additionally, given the increasing numbers of adults retaining their natural teeth, interventions designed to assist individuals maintaining healthy teeth becomes more critical.

\section{References}

1. Sanders AE, Slade GD, Carter KD, Stewart JF. Trends in prevalence of complete tooth loss among Australians, 1979-2002. Aust N Z J Public Health 2004;28:549-54.

2. Starr JM, Hall R. Predictors and correlates of edentulism in healthy older people. Curr Opin Clin Nutr Metab Care 2010;13:19-23.

3. Slade GD, Spencer AJ. Social impact of oral conditions among older adults. Aust Dent J 1994;39:35864.

4. Nowjack-Raymer RE, Sheiham A. Association of edentulism and diet and nutrition in US adults. J Dent Res 2003;82:123-6.

5. Kelly M, Steele J, Nuttall N, Bradnock G, Morris J, Nunn J et al. Adult Dental Health Survey Oral Health in the United Kingdom 1998. London, UK: Office for National Statistics, The Stationery Office; 2000.

6. Cunha-Cruz J, Hujoel PP, Nadanovsky P. Secular trends in socio-economic disparities in edentulism: USA, 1972-2001. J Dent Res 2007;86:131-6.

7. Dye BA, Tan S, Smith V, Lewis BG, Barker LK, Thornton-Evans $\mathrm{G}$ et al. Trends in oral health status: United States, 1988-1994 and 1999-2004. Vital Health Stat 11 2007; Apr:1-92.

8. United States Department of Health and Human Services. Healthy People 2010: Understanding and Improving Health. Washington, DC: US Department of Health and Human Services: For sale by the US G.P.O., Supt. of Docs.; 2000.

9. Schoenborn CA, Heyman KM. Health characteristics of adults aged 55 years and over: United States, 2004-2007. Natl Health Stat Report 2009;8:1-31.

10. Wu B, Liang J, Plassman BL, Remle RC, Bai L. Disparities in oral health among White, Black and Hispanic elders: an examination of edentulism and dental caries. J Pub Health Dent. doi: 10.1111/j.17527325.2011.00273.x [Published Online First: 31 May 2011].

11. Indian Health Service. An Oral Health Survey of American Indian and Alaska Native Dental Patients: Findings, Regional Differences \& National Comparisons. Rockville, MD: US Department of Health and Human Services; 2001.

12. Holm-Pedersen P, Schultz-Larsen K, Christiansen N, Avlund K. Tooth loss and subsequent disability and mortality in old age. J Am Geriatr Soc 2008;56:429-35.

13. Adair SM, Bowen WH, Burt BA, Kumar JV, Levy SM, Pendrys DG et al. Recommendations for using fluoride to prevent and control dental caries in the United States. Morb Mortal Wkly Rep 2001;50:1-42.

14. Featherstone JD. Prevention and reversal of dental caries: role of low level fluoride. Community Dent Oral Epidemiol 1999;27:31-40.

15. Marthaler TM. Changes in dental caries 1953-2003. Caries Res 2004;38:173-81.

16. Weyant RJ. Seven systematic reviews confirm topical fluoride therapy is effective in preventing dental caries. J Evid Based Dent Pract 2004;4:129-35.

17. Eklund SA. Changing treatment patterns. J Am Dent Assoc 1999;130:1707-12.

18. Truman BI, Gooch BF, Sulemana I, Gift HC, Horowitz AM, Evans CA et al. Reviews of evidence on interventions to prevent dental caries, oral and 
pharyngeal cancers, and sports-related craniofacial injuries. Am J Prev Med 2002;23(1 Suppl):21-54.

19. Jones DB, Niendorff WJ, Broderick EB. A review of the oral health of American Indian and Alaska Native elders. J Public Health Dent 2000;60(Suppl 1):256-60.

20. Sekiguchi E, Guay AH, Brown LJ, Spangler TJ Jr. Improving the oral health of Alaska Natives. Am J Public Health 2005;95:769-73.

21. US Department of Health \& Human Service PHS. Healthy People 2010 Progress Review: Oral health. Rockville, MD: National Institute of Health, National Institute of Dental and Craniofacial Research; 2008.

22. Quandt SA, Chen H, Bell RA, Anderson AM, Savoca MR, Kohrman T et al. Disparities in oral health status between older adults in a multiethnic rural commu- nity: the rural nutrition and oral health study. J Am Geriatr Soc 2009;57:1369-75.

23. Kiyak HA, Kamoh A, Persson RE, Persson GR. Ethnicity and oral health in community-dwelling older adults. Gen Dent 2002;50:513-8.

24. Douglass CW, Berlin J, Tennstedt S. The validity of self-reported oral health status in the elderly. J Public Health Dent 1991;51:220-2.

25. Gilbert GH, Duncan RP, Kulley AM. Validity of selfreported tooth counts during a telephone screening interview. J Public Health Dent 1997;57:176-80.

26. Slack-Smith L, Lange A, Paley G, O'Grady M, French D, Short L. Oral health and access to dental care: a qualitative investigation among older people in the community. Gerodontology 2010;27(2):104-13. 